\title{
Salzborn, Samuel: Kollektive Unschuld. Die Abwehr der Shoah im deutschen Erinnern, 136 S., Hentrich\&Hentrich, Berlin und Leipzig 2020.
}

\author{
Horst-Alfred Heinrich \\ Online publiziert: 17 . September 2020 \\ (C) Der/die Autor(en) 2020
}

Darf der gesellschaftliche Umgang mit dem Erbe des Nationalsozialismus als „Erfolgsgeschichte“ interpretiert werden? Oder ,ist der Glaube an eine tatsächliche Aufarbeitung“ „die größte Lebenslüge der Bundesrepublik“ (S. 104)? Diese pessimistische Auffassung vertritt Samuel Salzborn in seinem jetzt erschienenen Band, in dem er sich mit - so der Untertitel - der „Abwehr der Shoah im deutschen Erinnern“ auseinandersetzt.

Für das anzuzeigende Buch hat der Autor bewusst die Form des Essays gewählt, weil sie ihm die Möglichkeit biete, die These pointiert zu vertreten, ,eine selbstkritische Aufarbeitung der Vergangenheit“" habe es in der Bundesrepublik ,nur rudimentär gegeben“. Stattdessen sei ,die deutsche Gesellschaft über die Jahrzehnte hinweg [...] zur Erinnerungsabwehrgemeinschaft [...] geworden“" (S. 6f.).

Für den Beleg seiner These nutzt Salzborn verschiedene Argumentationsstränge, die er in sechs Kapiteln vorträgt. Führt er zunächst die Konzepte von ,Schuld, Erinnerung und Abwehr" im Hinblick auf die nach 1945 beobachtbaren Reaktionen der deutschen Bevölkerung bezüglich des Nationalsozialismus ein, kommt dann die partielle Aufarbeitung gepaart mit einer generellen Selbstentlastung der Gesellschaft zur Sprache. Im Detail geht es hier um die äußerst fragwürdige juristische Praxis der Nichtahndung der zwischen 1933 und 1945 verübten Verbrechen wie auch um die durch die Politik betriebene Integration der vielen Täter in Gesellschaft und Staatsapparat. Weiterhin zeichnet Salzborn die Trends der letzten 70 Jahre im Kulturbereich nach und fragt, inwieweit und wie sich Vertreter_innen speziell in Literatur und Film mit der Shoah auseinandersetzten. Dabei offenbaren sich Wellenbewegungen, die zwischen Anerkennung und Abwehr oszillierten. Als kritisch sei seit Beginn der 2000er Jahre die Tendenz zu beobachten, mit der Deutsche erneut wieder als Opfer stilisiert würden.

Im vierten Kapitel skizziert Salzborn unter Rückgriff auf die Ergebnisse einer Reihe von Umfragestudien die Verbreitung antisemitischer Tendenzen in der Bevölkerung und stellt zugleich einen Bezug zu den Diskursen über die Bewertung des

H.-A. Heinrich $(\bowtie)$

Universität Passau, Passau, Deutschland

E-Mail: Horst-Alfred.Heinrich@uni-passau.de 
Nationalsozialismus her. Dabei weist er sehr zurecht auf den Perspektivwechsel hin, der in der Öffentlichkeit mit der Paulskirchenrede von Martin Walser Fahrt aufnahm.

In den letzten beiden Kapiteln liefert der Autor weitere Argumente dafür, wie die Shoah mehr und mehr dethematisiert und dafür die deutsche Mehrheitsbevölkerung zum Opfer des Weltkrieges mit seinen Folgen und damit eben auch in der Gegenwart stilisiert wird. Diese Gedanken bringt er abschließend mit der zunehmenden Attraktivität der AfD wie auch rechtsextremer Bewegungen in Zusammenhang. Es dürfte unstrittig sein, dass deren Vertreter_innen die deutsche Vergangenheit diskursiv ,bereinigen ' wollen, um die eigenen Vorstellungen von einer ethnisch homogenen Volksgemeinschaft langfristig durchsetzen zu können.

Das Resümee des Rezensenten fällt nach dem Lesen etwas zwiegespalten aus. Wenn unter Rückgriff auf Wolfgang Hegener das „kollektiv getragene und komplizenhafte Verschweigen der Verbrechen durch weite Teile der Nachkriegsgesellschaft" (S. 105) als wirkmächtig bis in die Gegenwart beschrieben wird, ein Sachverhalt, den die aufgeklärten Eliten nicht wahrhaben wollten, darf man schon die Frage stellen, ob die Gegenwart damit korrekt beschrieben ist. Ist die „selbstreflexive Annäherung [an die verbrecherische Geschichte des Nationalsozialismus; HAH] bis heute nur das Anliegen einer kleinen, linksliberalen Elite in Politik und Öffentlichkeit" (S. 104), was keinen Widerhall in der deutschen Gesellschaft gefunden habe? Sind die vielen Graswurzelinitiativen vor Ort von den Geschichtswerkstätten bis hin zu Stolpersteinverlegungen damit als irrelevant einzuschätzen? Und wenn die Aufarbeitungsbemühungen als „größte Lebenslüge der Bundesrepublik“ bezeichnet werden (ebd.), stellt sich der Eindruck einer fehlenden Trennung zwischen Individual- und Gesellschaftsebene ein. Salzborn erwähnt unbestritten die Erfolge wie die Einführung des Gedenktags am 27. Januar oder die Errichtung des Denkmals für die ermordeten Juden Europas, doch nimmt er solche Hinweise an anderer Stelle wieder zurück. Diese Aussage gilt umso mehr, als die letzten Seiten des Bandes den von der AfD ausgehenden öffentlichen Diskurs der politischen Eliten als Bestätigung für die Schuldabwehr im deutschen Erinnern ins Zentrum rücken und ihm ein entsprechend starkes Gewicht geben.

Neben diesen kritischen Anmerkungen soll aber deutlich werden, dass Salzborns Essay neue Denkanstöße liefert. Ganz grundsätzlich stellt sich die Frage, ob die Kategorien von Schuld und Unschuld auf der individuellen Ebene hilfreiche Erklärungen liefern können. Wie Salzborn unter Rückgriff auf eine breite Literatur aus mehr als den letzten 50 Jahren zeigt, und wie Harald Welzer, Sabine Moller und Karoline Tschuggnall („Opa war kein Nazi“, 2002) mit einer Umfrage belegen konnten, geht die große Mehrheit junger Leute heute davon aus, in ihrer Familie habe man Widerstand geleistet. So absurd der Umfang des erinnerten persönlichen Widerstandes auch ist, verdeutlicht die Zahl doch die Brüche in den familialen und damit den sozialen Identitäten dieser jungen Menschen. Die nach wie vor offene Forschungsfrage ist danach, wie wir uns mit dem Wissen um die Schuld der Eltern und Großeltern eine positive soziale Identität verschaffen können, die wir Henri Tajfel und John Turner (Social identity theory, 1986) folgend - als Menschen benötigen.

Ungeachtet dieser Einwände bleibt festzuhalten, dass mit dem rezensierten Taschenbuch gerade für die politische Bildung ein wichtiger Baustein in der gegen- 
wärtigen Diskussion um die Gefährdung unserer Demokratie durch den Rechtsextremismus geliefert wird. Salzborn legt eine breit angelegte Darstellung des Diskurses zur Aufarbeitung der nationalsozialistischen Vergangenheit vor und bezieht einen klaren Standpunkt gegen Tendenzen, diesen Teil der Geschichte zu ,entsorgen'.

Funding Open Access funding provided by Projekt DEAL.

Open Access Dieser Artikel wird unter der Creative Commons Namensnennung 4.0 International Lizenz veröffentlicht, welche die Nutzung, Vervielfältigung, Bearbeitung, Verbreitung und Wiedergabe in jeglichem Medium und Format erlaubt, sofern Sie den/die ursprünglichen Autor(en) und die Quelle ordnungsgemäß nennen, einen Link zur Creative Commons Lizenz beifügen und angeben, ob Änderungen vorgenommen wurden.

Die in diesem Artikel enthaltenen Bilder und sonstiges Drittmaterial unterliegen ebenfalls der genannten Creative Commons Lizenz, sofern sich aus der Abbildungslegende nichts anderes ergibt. Sofern das betreffende Material nicht unter der genannten Creative Commons Lizenz steht und die betreffende Handlung nicht nach gesetzlichen Vorschriften erlaubt ist, ist für die oben aufgeführten Weiterverwendungen des Materials die Einwilligung des jeweiligen Rechteinhabers einzuholen.

Weitere Details zur Lizenz entnehmen Sie bitte der Lizenzinformation auf http://creativecommons.org/ licenses/by/4.0/deed.de.

Pfahl-Traughber, Armin: Rechtsextremismus in Deutschland. Eine kritische Bestandsaufnahme, 367 S., Springer VS, Wiesbaden 2019. Röpke, Andrea/Speit, Andreas: Völkische Landnahme. Alte Sippen, junge Siedler, rechte Ökos, 208 S., Links, Berlin 22019.

\section{Johannes Klotz}

Online publiziert: 29. Juli 2020

(C) Gesellschaft zur wissenschaftlichen Förderung politischer Literatur e.V. and the Author(s) 2020

Der Autor Armin Pfahl-Traughber nimmt in seiner „kritischen Bestandsaufnahme“ des aktuellen Rechtsextremismus Bezug auf sein 2014 erschienenes Buch zum Linksextremismus. Sie könne ,als entsprechender Blick auf die andere Seite ge-

J. Klotz $(\bowtie)$

Breisach am Rhein, Deutschland

E-Mail: dr.johannesk@gmail.com 